\title{
A Mediation Information System to Help to Coordinate the Response to a Crisis
}

\author{
Sébastien Truptil, Frédérick Bénaben, and Hervé Pingaud \\ Université de Toulouse - Mines Albi, France \\ \{truptil, benaben, pingaud\} @mines-albi.fr
}

\begin{abstract}
In a crisis situation, several heterogeneous actors have to work together in a hurry to solve or at least reduce the crisis. Their coordination and the transfer of information between them in such context are a crucial point. Unfortunately, lacks of coordination and collaboration have often been underlined as a major weakness of humanitarian crisis response. To help the whole of actors to work together, The ISyCri project aims to provide an information system. This system must be in charge of (i) information exchange, (ii) services sharing and (iii) behavior orchestration. Nevertheless, a crisis is by definition an evolutionary phenomenon. So a panel control is presented to help to detect when it is necessary to change the system because of the evolution of the situation. This paper aims to present, through an example, the approach of system creation and the control panel.
\end{abstract}

Keywords: interoperability, crisis management, evolution, information systems.

\section{Introduction}

According to [1], [2] and [3], a crisis is an abnormal situation which occurs suddenly and impacts a subpart of the world with unacceptable consequences. Such a break into the continuous state of the considered subpart of the world implies to deal with the crisis management through a dedicated set of stakeholders in charge of the crisis response. According to [2], [4], [5], crisis management is divided in four main parts:

1. Mitigation: this step aims at addressing the proactive social component of emergencies. This includes laws and mechanisms that reduce the vulnerability of the population and increases their resilience.

2. Preparedness: this step aims at defining the actors' abilities and preparing the means for crisis response. The definition of actors' abilities is made thanks to the analysis of past experience.

3. Response: once the crisis appears, several heterogeneous actors have to work simultaneously in a hurry with the global aim of crisis reduction.

4. Rehabilitation: this step aims to restore the (eco)system after the crisis. For example, rebuild buildings, schools...

The ISyCri project [6] focuses only on the response step of crisis management. Indeed, the global objective of the project is to help the whole heads of organizations 
in charge of the situation (in the following, we will use the terms: crisis cell), to deduce, on the base of preparedness results, a crisis response.

The crisis response concerns two kinds of actors. First, there are actors on the field, using their specific abilities (to perform activities such as evacuating injured persons, fixing a road, etc.). Second, there are heads of these actors on the field. Each head is in charge of, first the activities of operational actors on the field, and second, the exchange of information between the field and the crisis cell (report and instructions). Furthermore, even if actors share the same global goal, they do not tackle the same issue. The result of this point is the following: As explained in [7], the communication and the exchange of information inside the crisis cell is not sufficient enough to give each member the global view of the crisis and to help them to coordinate their activities. Nowadays, this lack of coordination and collaboration has been underlined as a major weakness of crisis response. This is why the ISyCri project focuses on the crisis cell and on the question of coordination and exchange of information between the stakeholders, inside the crisis cell.

The point is the following: considering that the stakeholders of the crisis response have the competences and the procedures to deal with their own mission, how is it possible to help to manage, to coordinate and to synchronize this set of operational experts of the crisis cell? Then this article aims at presenting the MIS deduction approach through an example. This approach is used to (help to) define, at the beginning of the crisis, the appropriate collaborative behavior through a collaborative process model. Nevertheless, because crisis is by definition a dynamic phenomenon, the crisis response has to be flexible. So the last part of this article aims at presenting the modification of the MIS according to the evolutions.

\section{Overview of ISyCri Project}

When a crisis occurs, several stakeholders are involved simultaneously to solve (or at least to control) the situation. The question is then: "How to provide this set of heterogeneous actors with the ability to organize and coordinate their actions in an optimal manner". Depending on the type, the size and the location of the crisis, this organization may be more or less formalized. The first hypothesis of this research work is that this collaborative organization is based on a crisis management cell, which may be seen as a decision center. The question then becomes: "how to provide the crisis cell with the ability to organize and coordinate the actions of heterogeneous stakeholders in an optimal manner".

Interoperability is defined in [8] by the European network of excellence InterOp as "the ability of a system or a product to work with other systems or products without special effort from the customer or user". It is also defined in [9] as "the ability of systems, natively independent, to interact in order to build harmonious and intentional collaborative behaviors without modifying deeply their individual structure or behavior". Considering these definitions we propose to consider that the previous main question is, at this point, the following: "how to support the interoperability of actors into the crisis cell". 


\subsection{Ideas and Tracks to Answer the Main Question}

An important point is that the members of the crisis cell have to communicate with their operational resources on the crisis field through their own channels. Another assumption of this article is that members of the crisis cell are able to use these specific means of communication to receive incoming reports and to send outgoing instructions. However, the central issue is that the members of the crisis cell are not used to exchange information between each other. If they expect to organize and coordinate their actions, they need to find a way to support their exchange of information into the crisis cell. This characteristic matches the framework proposed in [10] and so the proposed conceptual solution could be a Mediation Information System (MIS) dedicated to support interoperability.

To be useful for the crisis response, the MIS has to be perfectly relevant for the situation. That is why, the MIS deduction approach, start by deducing a collaborative process from the crisis information and the description of each stakeholder.

\subsection{Overview of MIS Deduction Approach}

The MIS deduction approach is divided into three main steps, corresponding exactly with the model-driven architecture defined by OMG [12]. First, a collaborative process deduction, explained in [13], from the crisis information and the stakeholders' information provides the representation of the coordination between the several stakeholders. This collaborative process is the CIM (computer independent model) of this approach. Second, a logical architecture of MIS, corresponding to the PIM (platform independent model), is build thanks to a transformation described in [14]. Third, another transformation is used to configure the technical deployment of MIS, corresponding to the PSM (platform specific model).

The collaborative process represents the collaboration while the last two steps of this approach are automatically executed. Consequently, we choose to illustrate only the first step through an example based on an exercise of crisis management realized by French authorities. This example was chosen because we witnessed the whole exercise, but this approach is also experimented in humanitarian crisis context.

The scenario is the following: "Policemen were informed that an accident occurred between a tanker and a train. A cloud of escaping products was created and people of railway station felt sick."

The model, represented by figure 1 , is the crisis model of this example that summarizes all the information of the crisis, as the "collision" event, the risks of explosion and contamination, the fact that some people felt sick.

Once this knowledge gathered, it is injected into an ontology already embedding information concerning partners' abilities. Then deduction rules, explained in [13] are executed to extract the stakeholders' abilities, which could be used to reduce one (or several) problem(s) of the crisis. Then to deduce the order of stakeholders' abilities execution, people in charge of crisis management must answer provide two choices: The priority ranking of identified problems and their selection among the set of selected abilities. In this example, the priority ranking is the following: (1) Explosion risk, (2) Contamination risk, (3) Sick people, (4) Panic risk. 


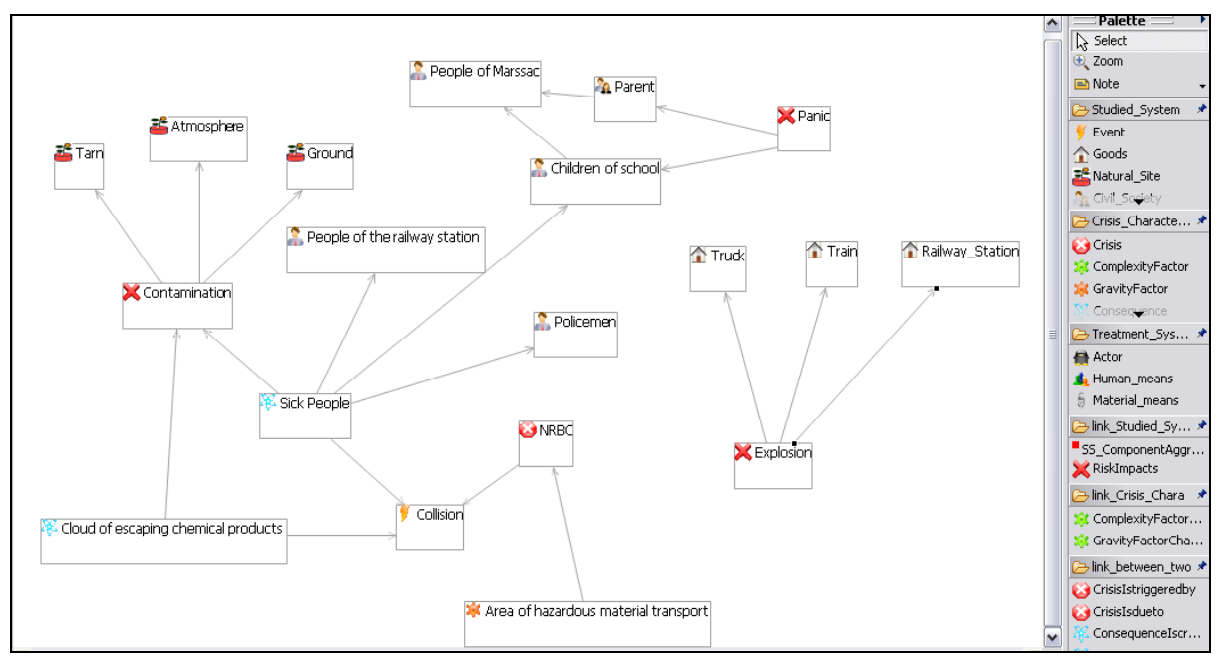

Fig. 1. Example of crisis model

Then abilities are selected (the partner in charge of the service is in brackets):

- Explosion risk: FightExplosion (Firemen), SetSecurityPerimeter (Policemen).

- Contamination risk: RescuePeople (Firemen), MaintainPerimeter (Policemen), SetMedicalPost (Red Cross).

- $\quad$ Sick people: BringPeopleToMedicalPost (Red Cross).

- Panic risk: Care People (EMS).

This set of services and the priority ranking of problems are the basic elements of the collaborative process deduction (figure 2) as explained in [13].

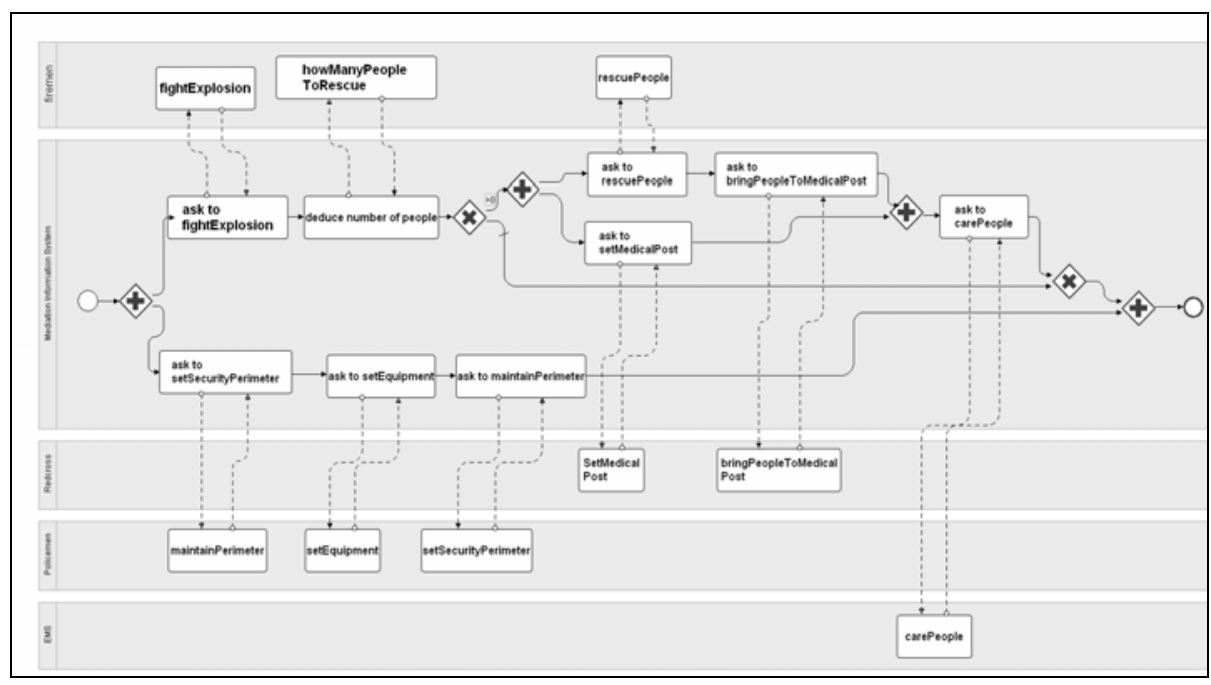

Fig. 2. Collaborative process 


\section{Limits of This Approach in Humanitarian Crisis}

In humanitarian crisis situation, the crisis cell is generally geographically disseminated. Consequently, the creation of an overall view, represented by the crisis model needs a tool to help the set of stakeholders to build it together. This tool has to collect all the information about the situation and then modify the crisis model according to them. We are currently working on tools, dedicated to the stakeholders IS, to transfer such modification. At this point, just one tool has been built to ensure the collect of information: EarlyWarning. This is a specific service that was build to collect information about the situation. For this, each stakeholder has its own interface of EarlyWarning that allows it to share information. Depending on this information, the crisis model is modified either with the creation or deletion of problems.

At any moment, the design approach, explained in section 2 , can be executed to build a MIS adapted to the crisis model and the stakeholders' abilities. Unfortunately, new information about the situation and/or stakeholders could feasibly render MIS obsolete at any moment. So in our work we believe that it is a better way to build a MIS and then modify it than to wait after the whole information about the situation. This point of view implies that the MIS has to evolve according to the situation. In the following part of this article, we explain how our tool allows to detect and take into account the potential evolutions of the situation.

\subsection{Possible Evolutions and Their Impacts on MIS Deduction Approach}

Crisis situation is by definition an evolutionary phenomenon. So, the crisis response has to be able to adapt depending on evolutions. In [9], the author proposes a classification of evolutions, which can occur in context of collaboration between several heterogeneous organizations. In crisis management context, this classification can be adapted as follows: (i) the crisis itself changes (several earthquakes successively), (ii) the set of organizations in charge of crisis response evolves during the response (an organization quits the crisis field) (iii) dysfunction can append during the crisis response (restarting of a fire).

MIS, and collaborative process has to be maintained in order to correspond exactly to the situation, depending on the kind of evolutions. Indeed, (i) if the crisis itself evolves, then the collaborative process is out-of-date, whereas, (ii) if the crisis situation does not evolve but the whole of stakeholders changes, then, the collaborative process may just need a small adaptation, (iii) finally, if a dysfunction happens during the crisis response, the collaborative process may be partially reexecuted, but the situation or the set of stakeholders may also require an adaptation.

Therefore, when an evolution occurs, the MIS design process can be restarted at several levels to obtain a MIS, specifically dedicated to the current situation. Nevertheless, it is necessary to ensure that what has already been done is taken into account. For this, another tool has been added: mediation services.

At the beginning, this tool was created to realize information's transformations from one service of a stakeholder to another. Consequently, before each service, a data transformation is realized, currently based on a static correspondence table. By this way, we obtained a new functionality, which send the state of the service (waiting, started, finished or execution fault). This functionality allows to follow the collaborative process and to know status of any service at any time. 
In crisis context, we believe that it is not possible to automatically decide if MIS has to be modified. So, we try to help the crisis cell to take that decision by providing, a control panel. It aggregates all the information send by EarlyWarning and mediation services and helps the crisis cell to detect evolutions.

\subsection{How to Detect the Several Kinds of Evolutions}

In this part, a control panel is proposed to help to detect the evolutions. This control panel, represented in figure 3, is composed of four tables. The two upper tables refer to risks (potential problem) and consequences (actual problem), of the crisis. The others refer to the crisis response (Actors state and Services state).

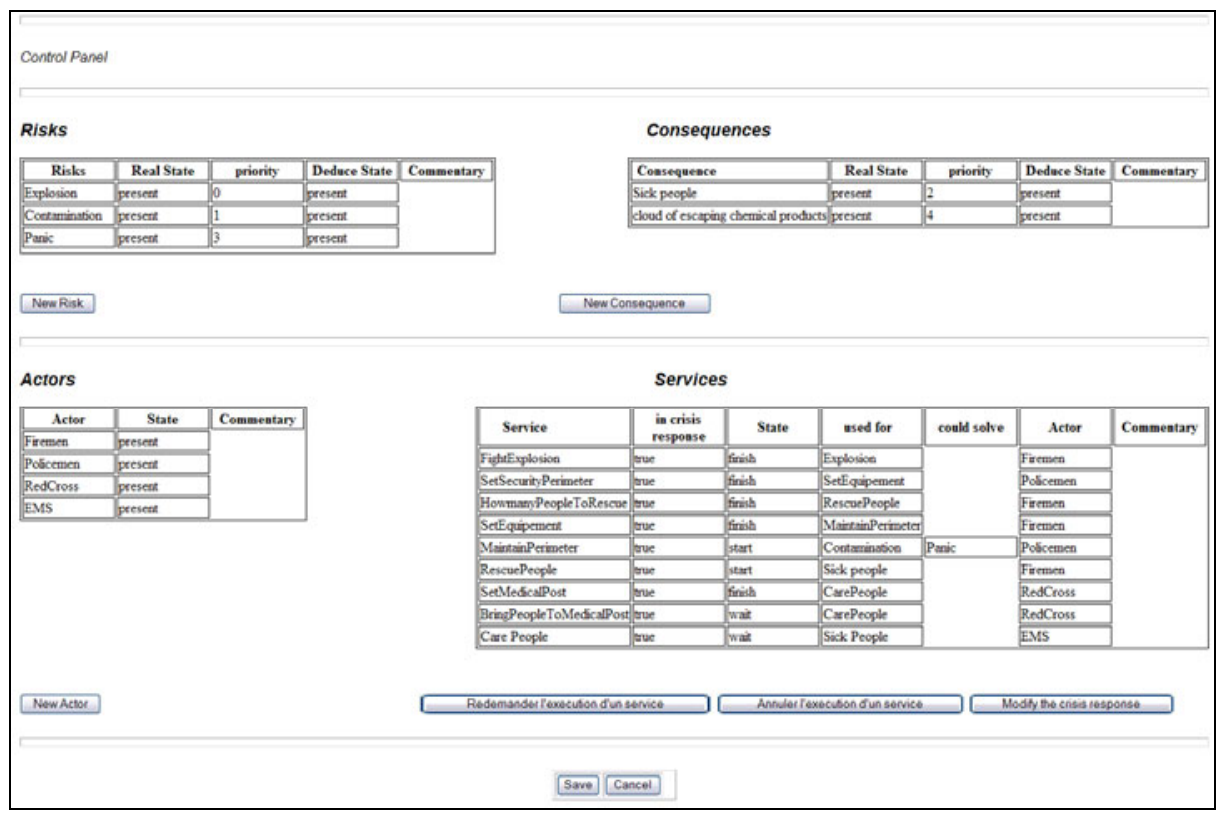

Fig. 3. Panel control uses to detect the evolutions

The two first tables have the same use. They summarize the whole of characteristics of the crisis and their treatment priority. They inform on the difference between the real state and the deduced state of crisis characteristics. The real state corresponds to the real situation thanks to information coming from the field. The deduced state is inferred from the progress of the crisis response. When all the services that have been specifically chosen to reduce the crisis characteristic A have been executed, the deduced state of that characteristic A is turn into solved.

The two others tables (Actors and Services) are used to draw an overview of the crisis response and to detect dysfunctions or changes into the crisis cell composition. Indeed, the service table inform on the collaborative process execution, especially on the service state (waiting, started, finished or execution fault) whereas the actor table inform on the crisis cell composition, and each partner's state (present, wait, leave). 
Based on the supervision of these tables, four kinds of reaction are available:

- If a new information appears in one of the two first tables (Risk and Consequence), the crisis cell has to change the crisis model and launch again the whole MIS deduction approach (because a critical evolution of the situation has just appeared).

- If there is a difference between the deduced state and the real state of one risk or consequence, the crisis cell has to change the crisis response and the MIS because at least one dysfunction occurred. In this case, the crisis cell has several choices: (i) restart a service. (ii) Launch again the MIS deduction approach with the same crisis model, with other ranking choices or service affectation choices.

- If there is a problem during the execution of service (service state $=$ fault), the crisis cell has to change the crisis response according to the same options than the ones presented at the last point.

- If the state of a partner changes, because it quits or joins, the crisis cell may also have to change the response according to the same options than the ones presented at the last point.

\section{Conclusion and Future Work}

The presented works aims at providing a mediation information system able to support the interoperability between information systems in a crisis context. The design of the MIS is based on the following assumptions: (i) the crisis management is assumed by a crisis cell, (ii) each stakeholder of the crisis cell is able to ensure the communication with its own resources on the operational field and finally, (iii) each stakeholder of the crisis cell is able to propose a set of services reflecting its concrete operational abilities. The two first ones could not be changed for the moment, but concerning the third one an interesting perspective may be the support of service design. This track concerns the assisted conception of technical services, according to interfaces patterns and business services analysis.

One obvious weak point of this work concerns the fact that a single collaborative process may be a too poor model to describe the crisis response. It could be better to get several processes, which could cover several levels of process (decisional, operational and support).

Finally the use of the panel control provides the crisis cell with an overview of the crisis response and a way to detect the possible evolutions. Today, on the occurrence of any evolution, the crisis cell modifies on its own the crisis response (process and/or activities). Thus, automatic modifications can be deduced from specific events. These automatic modifications could be a strong improvement for the crisis cell management.

\section{References}

1. Devlin, E.: Crisis Management Planning And Execution, p. 528 (2006)

2. Tomasini, R.: Van Wassenhove, L.: Humanitarian logistics INSEAD (2009) 
3. Lagadec, P.: La gestion des crises. In: Ediscience International, p. 326 (1992)

4. Atlay, N., Green, W.: OR/MS research in disaster operations management. European Journal of Operational Research 175, 175-475 (2005)

5. Beamon, B.M., Kotleba, S.A.: Inventory modelling for complex emergencies in humanitarian relief operations. International Journal of Logistics: Research and Applications 9, 1-18

6. Truptil, S., Bénaben, F., Couget, P., Lauras, M., Chapurlat, V., Pingaud, H.: Interoperability of Information Systems in Crisis Management: Crisis Modeling and Metamodeling. In: IESA 2008. Springer, Germany (2008)

7. Hansen-Glize, C.: de la maîtrise des crises. Techniques de l'ingénieur, France (2008)

8. Konstantas, D., Bourrières, J.P., Léonard, M., Boudjlida, N.: Interoperability of Entreprise Software and Applications. Springer, Genoa (2005)

9. Pingaud, H.: Rationalité du développement de l'interopérabilité dans les organisations. In: Management des technologies organisationnelles, pp. 19-30. Presses de l'Ecole des Mines de Paris, France (2009)

10. Wiederhold, G., Genesereth, M.: The conceptual basis for mediation services. IEEE Expert: Intelligent Systems and their Applications 12(5), 38-47 (1997)

11. Benguria, G., Larrucea, X., Elveseater, B., Neple, T., Beardsmore, A., Friess, M.: A Platform Independent Model for Service Oriented Architectures. In: IESA 2006, Bordeaux, France, pp. 407-416. Springer, Heidelberg (2006)

12. OMG, MDA Guide Version 1.0.1, omg/2003-06-01 (2003)

13. Truptil, S., Bénaben, F., Pingaud, H.: Collaborative process deduction to help the crisis cell emerging ecosystem to coordinate the crisis response. In: IEEE DEST 2010, Dubai (2010) (accepted paper to be published)

14. Touzi, J., Bénaben, F., Pingaud, H., Lorré, J.P.: A Model-Driven approach for Collaborative Service-Oriented Architecture design. International Journal of Production Economics 121(1), 5-20 (2009) 